\title{
A mathematical model of saccadic reaction time as a function of the fixation point brightness gain
}

\author{
Antonio Diaz-Tula ${ }^{1}$. Carlos H. Morimoto ${ }^{1} \cdot$ Ronald D. Ranvaud $^{2}$
}

Published online: 12 May 2015

(C) The Psychonomic Society, Inc. 2015

\begin{abstract}
The gap effect refers to a reduction in saccadic reaction time $(S R T)$ to an eccentric target, when the fixation point is removed before the target onset. Though it is known that the gap effect peaks when the fixation point is offset about $200 \mathrm{~ms}$ before the onset of the eccentric target, it is unknown how this effect is modulated by stimulus variations. In this paper, we propose and investigate a model of saccadic reaction time as a function of the fixation point brightness gain. The brightness gain is defined as the ratio of the final and initial intensities of the stimulus. We have conducted a typical gap effect experiment with 15 participants, where the brightness of the fixation point was manipulated under four conditions and two gap intervals, at the same time and $200 \mathrm{~ms}$ before the onset of the eccentric target. The conditions included removing the fixation point (offset), leaving it with constant brightness (overlap), reducing, and increasing its brightness (lower and higher brightness condi-
\end{abstract}

Antonio Diaz-Tula

diaztula@ime.usp.br

Carlos H. Morimoto

hitoshi@ime.usp.br

Ronald D. Ranvaud

ranvaud@usp.br

1 Department of Computer Science, Institute of Mathematics and Statistics, University of São Paulo, Rua do Matão 1010, São Paulo, SP, Brazil

2 Department of Physiology and Biophysics, Institute of Biomedical Sciences, University of São Paulo, Av. Prof. Lineu Prestes, 1524, São Paulo, SP, Brazil tions). Experimental data showed a significant gap effect in the offset and lower brightness conditions when compared to the overlap condition. On the other hand, the $S R T$ was significantly longer for the higher brightness condition than the $S R T$ for the overlap condition. Linear regression analysis using ten values of brightness gain shows that our model fits the data well for the 0 - and 200-ms gap, with a coefficient of determination of .89 and .94 , respectively.

Keywords Gap effect - Saccadic reaction time - Perceptual changes - Bottom-up attention - Attentional engagement modulation

\section{Introduction}

The gap effect, a phenomenon first described by Saslow (1967), corresponds to a reduction in saccadic reaction time $(S R T)$ when the fixation point is removed some time before the onset of the eccentric target, i.e., when there is a temporal "gap" between the removal of the fixation point and the onset of the eccentric target. The reaction time improvement relative to the overlap condition, where the fixation point remains the same before and after the onset of the eccentric target, typically peaks for gap values around $200 \mathrm{~ms}$.

Several studies have shown that there are different components that can contribute to a reduction of the saccadic latency. Recent studies seem to agree that the dominant components are due to the attentional disengagement (or release) and the fixation offset effect (FOE).

The attentional disengagement theory is based on the attention model of Posner and Petersen (1990). According to this model, to switch between two visual targets, attention first needs to be disengaged from its current focus, then shifted to the new location, and finally must be re-engaged 
on the visual target. Fisher and Breitmeyer (1987) proposed that the offset of the fixation point produces a disengagement of attention that reduces the SRT. Subsequent studies have supported this theory (Jin and Reeves, 2009; Mackeben and Nakayama 1993; Pratt et al., 2006).

The fixation offset effect theory is corroborated by the study of Munoz and Wurtz (1992). From experiments carried out in monkeys, they showed that while fixating a visual stimulus, neurons in the superior colliculus discharge in a tonic manner and inhibit the execution of saccades. During a saccade, these neurons pause their activity permitting the saccadic movement. Several studies (Dorris and Munoz, 1995; Kingstone \& Klein, 1993; Reuter-Lorenz et al., 1995) have proposed that the offset of the fixation point pauses the activity of the fixation neurons during the gap interval, producing an oculomotor release that facilitates the saccade and hence reduces the latency.

Typical gap effect experiments manipulate visual properties of the fixation point or of the eccentric target. For example, Reuter-Lorenz et al. (1991) studied the effect of two luminance levels for the eccentric target, one bright and one dim. They found that brighter eccentric targets produce shorter reactions times, and that this effect is additive with the $S R T$ reduction due to the offset of the fixation point. Bell et al. (2006) also found that brighter targets produce shorter SRTs in monkeys, concluding that brighter targets are processed faster by the visual system.

Reulen (1984) measured SRTs for three different levels of the fixation point brightness (high, medium, and low), while keeping the intensity of the eccentric target constant (always high brightness). The results of Reulen's experiments showed that the mean $S R T$ was longer for lower brightness values of the fixation point. Reulen also concluded that only a significant decrease in the brightness of the fixation stimulus shortens the $S R T$, but the magnitude of the decrease was not defined.

Instead of brightness, Pratt et al. (2000) manipulated the area of the fixation point to estimate the contribution of visual warning and FOE to the gap effect. Their study included an auditory warning in half of the trials. The authors found a gap effect of $67 \mathrm{~ms}$ in the absence of the warning tone, and $40 \mathrm{~ms}$ when the warning tone was present, thus confirming that alertness modulates the gap effect.

Jin and Reeves (2009) evaluated several theories of the gap effect and concluded that attentional release has a major impact on the $S R T$ reduction, while oculomotor release has a smaller but significant effect. The authors included in their experiments the dim gap trial, where the luminance of the fixation point was reduced, and the white gap trial, where the luminance was increased. Although the dim and white gap trials were selected to provide equally salient warning signals, SRTs were shorter in the dim gap trial than in the white gap trial. The authors assumed that this difference was because dimming the fixation point sufficiently is similar to turning it off, hence releasing attention, whereas brightening it would hold attention. They also ran a finger-press reaction time experiment to foveal probe dots and confirmed that attention was held after the fixation point was brightened, and released when it was removed.

These results can be explained in terms of the bottom-up account of visual attention, according to which attentional allocation is driven by external factors such as stimuli salience produced by, for example, movement, contrast, and loudness (Awh et al., 2012). An example of the bottom-up control of attention can be found in the cueing paradigm, where subjects have to initially fixate a central stimulus and then search for a target that is presented at one of several possible locations (Fuchs \& Ansorge, 2012). A taskirrelevant, exogenous cue presented before the target onset facilitates the search task if the cue's location coincides with the future target location. This facilitation supports the idea that attention is attracted by the cue.

There is evidence that higher-contrast cues attract more attention than lower-contrast cues (Fuchs \& Ansorge, 2012; Fuller et al., 2009; Kean \& Lambert, 2003). Furthermore, Fuller et al. (2009) found that attention is modulated by cue contrast, concluding that although allocation of attention is automatic and unconscious, the attentional process is gradual rather than all-or-none.

In gap effect experiments where the task does not demand any specific conscious attentional behavior (Bell et al., 2006; Jin and Reeves, 2009; Pratt et al., 2000; Reuter-Lorenz et al., 1991), it is reasonable to consider that attention is mainly driven by the stimuli salience.

Considering the role of attention in the gap effect and the evidence from the cueing paradigm that bottom-up allocation of attention is modulated by cue contrast, we hypothesized that the attentional engagement component of the gap effect can be modulated by perceptual changes in the fixation point.

If this hypothesis is correct, we expect that the saccadic reaction time can be expressed by psychophysical laws such as Weber-Fechner's law (Fechner, 1860), since it is a perceptual response to physical stimuli. The Weber-Fechner law has been applied in saccadic latency models to encode the difference in luminance or contrast of a visual target, in situations where the target is present or not (Carpenter, 2004) or when its intensity changes from an initial to a final value (Taylor et al., 2006).

We developed a mathematical model that expresses the $S R T$ as a logarithmic function of the brightness gain (the ratio between the final and initial brightness of the fixation point). The significance of such a model is twofold. In practical terms, it permits quantifying the probable effect of different stimuli manipulation on $S R T$, without having to try each one, and also to estimate if a desired effect 
on $S R T$ is possible by manipulating stimuli. In theoretical terms it establishes a quantitative link between perception and action, based on Weber-Fechner's law (Fechner, 1860) which states that perceptual response is a logarithmic function of stimulus physical intensity.

The model is introduced in the next section, and is then tested in an experiment where the brightness of the fixation point was changed for several initial and final levels.

\section{Mathematical model for saccadic reaction time}

Let's assume that the initial luminance of the fixation point is $L_{\text {init }}$ and, some time (gap) before the onset of the eccentric target, it changes to a final value $L_{\text {final }}$. Let's assume also that the background where the stimuli are presented is black (luminance $0 \mathrm{~cd} / \mathrm{m}^{2}$ ), similar to previous gap effect experiments (Jin and Reeves, 2009; Pratt et al., 2000; Reuter-Lorenz et al., 1995, 1991), and that the ambient illumination is $L_{a}$. The intensity of the light that reaches the observer's eyes are $L_{\text {init }}+L_{a}$ and $L_{\text {final }}+L_{a}$ for the initial and final values of the fixation point, respectively.

Weber-Fechner's law (Fechner, 1860) states that the subjective sensation of a stimulus is proportional to the logarithm of its physical magnitude. According to this law, the subjective sensation produced by the initial and final brightness of the fixation point would be $\log \left(L_{i n i t}+L_{a}\right)$ and $\log \left(L_{\text {final }}+L_{a}\right)$, respectively. Hence, the subjective sensation difference produced by the brightness change of the fixation point can be expressed as:

$\log \left(\frac{L_{\text {final }}+L_{a}}{L_{\text {init }}+L_{a}}\right)$

It is noteworthy that the logarithm argument in Eq. 1 is independent of the units or representation used for the intensity of the fixation point. Because of that, we have named it the brightness gain $(B G)$. Using Eq. 1, we propose that the saccadic reaction time can be expressed mathematically by:

$S R T=\alpha \cdot \log (B G)+\beta$

where the values of $\alpha$ and $\beta$ are numerical constants determined empirically. We define $\alpha$ as the attentional engagement factor and represents the slope of the lin-log graph curve of $S R T$ by $\log (B G)$. A steep slope implies that the $S R T$ is very sensitive to variations of the brightness gain, while a more gradual slope (flat) implies that the reaction time will vary little even for large brightness variations. We define $\beta$ as the non-modulated $\boldsymbol{S R T}$ since it represents the saccadic reaction time measured without any visual manipulation of the fixation point (overlap condition). Observe that when the brightness gain is equal to $1, \log (B G)$ is zero and the parameter $\beta$ is equal to the $S R T$ observed in the overlap condition.
When the brightness gain is lower than $1, \log (B G)$ is negative and the attentional engagement is reduced, thus facilitating the saccade, i.e., the $S R T$ will be shorter than $\beta$. On the other hand, when the brightness gain is greater than $1, \log (B G)$ is positive and the attentional engagement is enhanced (the $S R T$ will be longer than $\beta$ ).

In order to validate our quantitative model of saccadic reaction time, we designed an experiment with several levels of initial and final brightness of the fixation point and two gap intervals that is described in the next section.

\section{Methods}

\section{Participants}

Sixteen people from the University of São Paulo participated in the experiment (three female) with ages ranging from 21 to 50 years old, average 28.6 ( \pm 8 ). All participants had normal or corrected to normal vision and seven of them had never used an eye tracker before. The research protocol was approved by the Ethics Board of the Institute of Biomedical Sciences of the University of São Paulo.

\section{Apparatus}

Eye movement data was collected using an SMI RED500 remote eye tracker with a sampling frequency of $500 \mathrm{~Hz}$. The eye tracker was placed at the bottom of a 22" LCD monitor used to present the visual stimuli. The brightness and contrast of the monitor were initially set to their maximum values $(100 \%)$, and the brightness of the visual stimuli was controlled by software during the experiment. The experiment was conducted in a room with regular fluorescent illumination, common in office spaces, with no direct sunlight as recommended by Holmqvist et al. (2011).

The eye tracker and monitor were positioned $60 \mathrm{~cm}$ from the participant's eyes as depicted in Fig. 1. A chin rest was used during the experiment to reduce head movements and

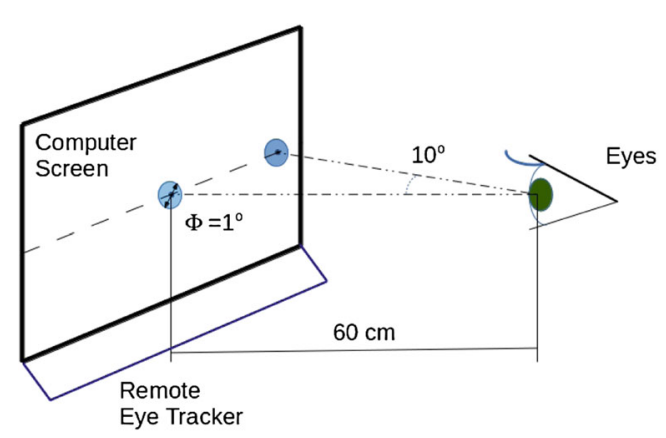

Fig. 1 Experimental setup showing the position of the participant's eyes, the monitor, and the eye tracker 
keep the eyes at approximately the same height as the center of the screen. Disks with a diameter of $1^{\circ}$ of visual angle were used as visual stimuli. A small dot was placed at the center of the disks to serve as target. The distance between the fixation point and the eccentric target corresponds to $10^{\circ}$ of visual angle.

\section{Design}

In order to study the effect of perceptual changes of the fixation point in attentional engagement, SRTs for several configurations with different values of brightness gain must be evaluated. The other independent variable in our experiment was the gap interval.

We defined three levels of initial brightness and four levels of final brightness of the fixation point. The initial brightness levels were high (100\% brightness of the monitor), medium (50\% of the maximum brightness of the monitor), and low (5\% of the maximum brightness of the monitor). The final brightness levels were the same high, medium, low, and zero ( $0 \%$ of the maximum brightness of the monitor). The gap values were 0 and $200 \mathrm{~ms}$.

The following four conditions were defined, each containing three manipulations of the fixation point. For the overlap condition the fixation point stays high $(\mathrm{HH})$, medium (MM), or low (LL). Observe that the notation used in parenthesis corresponds to the manipulation of the fixation point, i.e., $\mathrm{HH}$ implies that the fixation point started at $\mathrm{H}$ and there was no change. For the offset condition (fixation point disappears), the fixation point goes to zero, from high (HZ), medium (MZ), or low (LZ) intensities. For the lower brightness condition, the fixation point intensity decreases in one of three possible ways: HL, ML, or HM. Finally, for the higher brightness condition, the fixation point intensity increases in one of three possible ways, which are the opposite of the lower condition: LH, LM, and $\mathrm{MH}$.

A fully crossed design was adopted thus resulting in 4 (conditions) $\times 3$ (fixation point manipulations per condition $) \times 2$ (gap values $)=24$ configurations. Each configuration shall be referred to by a number ( 0 for zero gap, 2 for 200-ms gap), and two letters, as above, that define the fixation point manipulation. Thus, 2 HL refers to the configuration in which the fixation point changes for high to low intensity $200 \mathrm{~ms}$ before target onset.

The offset configurations served to validate our experiment by comparing with existing studies where the fixation point was removed. The overlap condition (brightness of the fixation point was constant) was used as the control. According to our model, all overlap configurations should elicit similar $S R T \mathrm{~s}$, since the brightness gain is always 1 , regardless of the fixation point brightness. For the lower brightness condition shorter SRTs than in the control condition are to be expected, because of the attentional release produced by dimming the fixation point. Finally, for the higher brightness condition SRTs would be expected to be longer than in all other conditions, since brightening the fixation point engage more attention.

In the experimental conditions described above, with exception of the overlap condition, the brightness change of the fixation point occurs at the same time or $200 \mathrm{~ms}$ before the target onset. This change acts as a top-down visual warning that permits the subject to prepare to make the saccade (Trappenberg et al., 2001). Since we wanted to estimate the effect of perceptual changes on attention for all conditions, including the overlap condition, a 1-kHz auditory warning was played $100 \mathrm{~ms}$ before the visual manipulation, similar to (Craig et al., 1999; Pratt et al., 2006, 2000; Reuter-Lorenz et al., 1995, 1991), so as to introduce a warning signal in all conditions.

Because the luminance intensity varies among monitors, to make our experiment replicable, the monitor was calibrated with a J1803 Tektronix LumaColor ${ }^{T M}$ Photometer. We manually annotated the photometer readings in $\mathrm{cd} / \mathrm{m}^{2}$ for equally spaced values of brightness levels at the center of the monitor, where the fixation point was shown during the experiment. The calibration showed that our monitor had a power luminance curve expressed by $y=249.9 x^{2.26}, R^{2}=$ .99, where $x$ is the brightness percent (expressed as a fraction, i.e., for a $100 \%$ brightness $x$ equals 1 ) and $y$ is the luminance in $\mathrm{cd} / \mathrm{m}^{2}$. The values of the levels high, medium, low, and zero were converted to $\mathrm{cd} / \mathrm{m}^{2}$, resulting in the values presented in Table 1.

The ambient luminance was computed by placing the photometer at approximately the same position of the participants' eyes, pointing towards the center of the monitor, which was turned off during this procedure. The ambient luminance was obtained after the photometer reading stabilized at $4 \mathrm{~cd} / \mathrm{m}^{2}$.

\section{Procedure}

Each participant was asked to initially look at the fixation point at the center of the screen until an eccentric target

Table 1 Conversion between brightness levels and luminance

\begin{tabular}{lll}
\hline Level & Brightness percent & Luminance $\left(\mathrm{cd} / \mathrm{m}^{2}\right)$ \\
\hline high & 100 & 249.9 \\
medium & 50 & 52.0 \\
low & 5 & 0.28 \\
zero & 0 & 0 \\
\hline
\end{tabular}

Brightness levels are expressed as percents of the maximum brightness of the monitor. 
appeared, and then look as fast as possible at the target, $10^{\circ}$ to the left or right-hand side of the fixation point. Participants repeated 20 times every possible configuration. From the 20 repetitions, the eccentric target appeared ten times to the left and ten times to the right of the central fixation point in random order. Therefore, each participant performed $20 \times 24=480$ trials. The order of the trials was randomly selected for each participant.
Each trial was composed of five phases as shown in Fig. 2: an initial fixation phase, followed by an auditory warning, fixation point manipulation, and gap periods, and finally the phase that started with the onset of the eccentric target. Observe that in some conditions the duration of a phase could be $0 \mathrm{~ms}$.

During the initial phase, the central stimulus was presented for a period uniformly distributed in the range 400 to

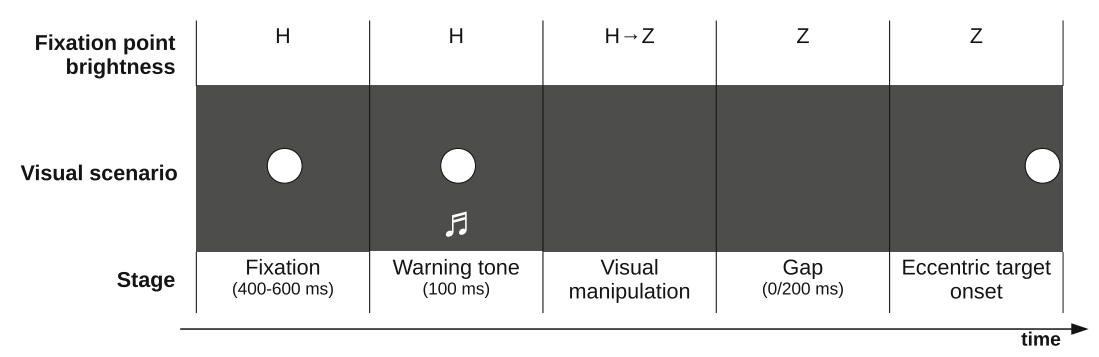

a) Offset condition trial where the brightness drops from high to zero, with a zero or $200 \mathrm{~ms}$ gap.

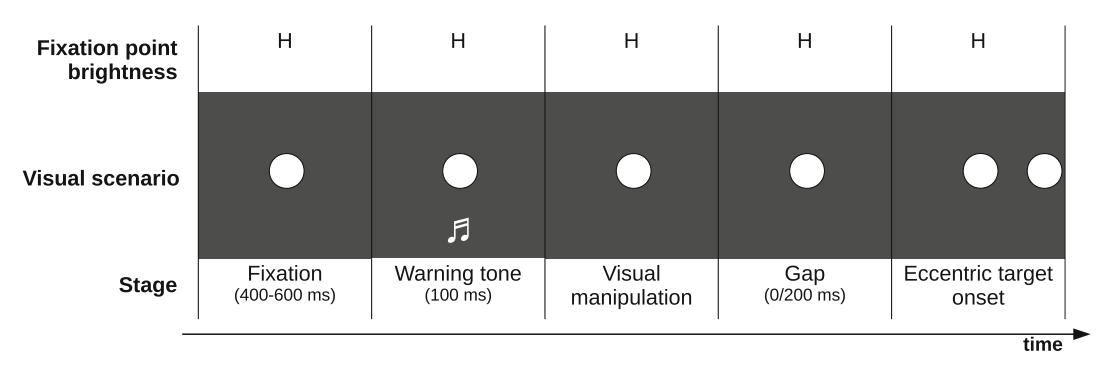

b) Overlap condition where the fixation point never changes (stays at high) throughout the trial.

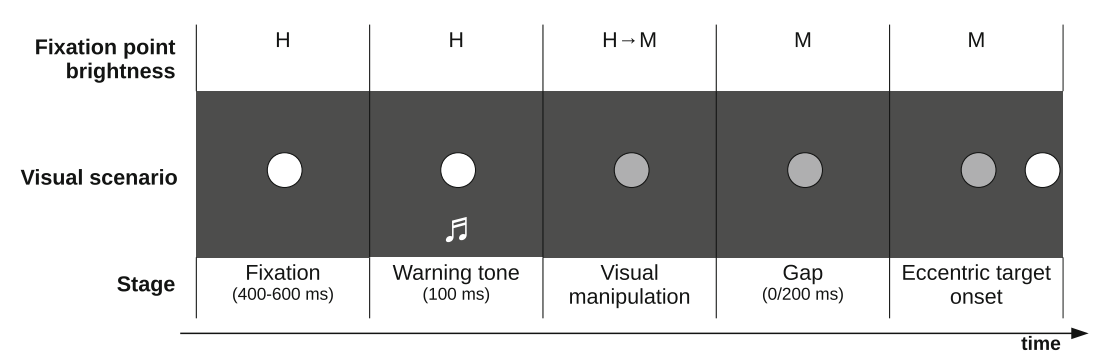

c) Lower brightness condition, where the final brightness of the fixation point is lower than its initial brightness (in this case from high to medium).

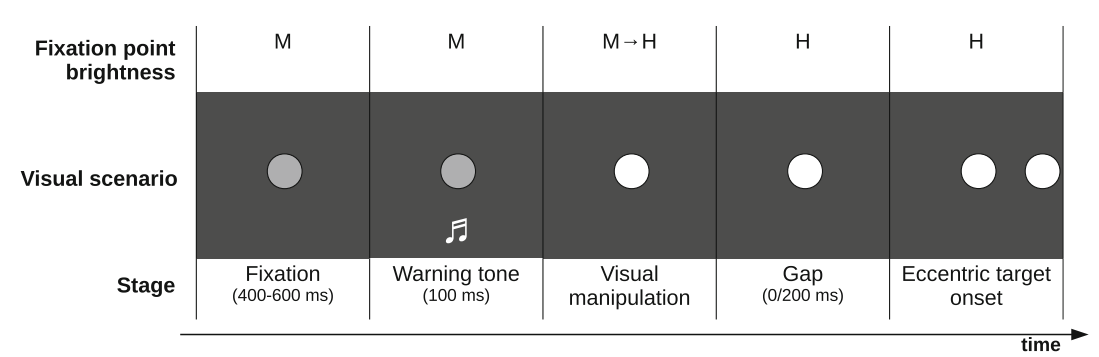

d) Higher brightness condition, where the brightness of the fixation point is increased (in this case from medium to high).

Fig. 2 Gap, overlap, lower brightness and higher brightness conditions used in our experiment 
$600 \mathrm{~ms}$, to avoid any learning/prediction bias in the results. A $1-\mathrm{kHz}$ warning tone that lasted $100 \mathrm{~ms}$ followed in the second phase. The visual manipulation of the fixation point occurred step-wise (i.e., in successive frames), at the end of the warning tone, so was a phase that lasted $0 \mathrm{~ms}$. In this phase, the fixation point might disappear (offset condition), remain with constant brightness (overlap condition), or have its brightness decreased (lower brightness condition) or increased (higher brightness condition). The fourth phase was the gap period ( 0 or $200 \mathrm{~ms}$ ), which preceded the final phase, in which the eccentric target appeared, always with brightness level high, at the left or right-hand side of the fixation point. Participants were asked to gaze at the eccentric target as fast as possible after it appeared, and to keep fixating until it disappeared.

Figure 2 shows a graphic representation of the four conditions used in the experiment. The first row shows an example of how the fixation point changes during an offset condition trial where the brightness drops from high to zero, with a 0 - or 200-ms gap. The second row shows the overlap condition, where the fixation point never changes (in this example stays at high) throughout the trial. Figure $2 \mathrm{c}$ shows an example of the lower brightness condition, where the final brightness of the fixation point is lower than its initial brightness. In this example, the fixation point goes from high to medium. The last row of Fig. 2 shows an example of the higher brightness condition, where the fixation point varies from medium to high.

\section{Data collection and processing}

Each participant visited our laboratory once and remained there for about $35 \mathrm{~min}$. Data gathering was divided into four sessions, each one having a duration of approximately $5 \mathrm{~min}$ with at least 5 min break between sessions. Each session was composed of ten sets of 12 trials each, and each set lasted about $30 \mathrm{~s}$. Participants were instructed to try not to blink during each set and they were free to rest their eyes between sets. Participants initiated each set by pressing a key. All participants received a brief introduction to the experiment and signed an informed consent form. After the introduction, the eye tracker was calibrated and participants were familiarized with the experiment through a short training session.

The software for controlling the experiment, i.e., manipulating the visual stimuli and recording the eye movements, was written using the Python programming language and the PsychoPy library (Peirce, 2009). During the experiment, the software was designed to identify some problems and try to correct them. When the data quality was degraded above a certain threshold by calibration drifts of the eye tracker, the software interrupted the execution of the current set of saccades, asked the participant to re-calibrate the eye tracker, and repeated the interrupted set at most once. The software computed fixations in real time using a dispersionbased algorithm (Salvucci and Goldberg, 2000) and was designed to detect situations where participants ended the fixation on the central stimulus before the onset of the eccentric target (anticipations). The software also detected blinks using information of the pupil diameter. When such anticipations or blinks were detected, the unsuccessful trial was appended to a list of trials that were repeated in a separate set (or sets) at the end of the session. To keep the sessions within reasonable duration, trials in the unsuccessful list were not repeated and, in case of a second problem, the trial was discarded.

Saccades were detected offline using a variation of the velocity-threshold fixation identification (I-VT) algorithm of Salvucci and Goldberg (2000). For each trial, the algorithm considered the midpoint of the left and right eye coordinates from the first $500 \mathrm{~ms}$ after the onset of the eccentric target. Before detecting saccades, eye tracker acquisitions were filtered to remove high-frequency noise using a moving average filter. Trials with more than three consecutive invalid acquisitions (as reported by the eye tracker) were discarded. Invalid acquisitions were substituted by the mean of a window of size 5 centered at the acquisition.

\section{Results}

We recorded a total of 7407 trials, including those that were repeated due to calibration drifts, blinks, and anticipations. In $0.4 \%$ of the total number of recorded trials, the saccade detection algorithm did not detect saccades, in $5.7 \%$ the saccade was not directed towards the eccentric target, $2.7 \%$ had blinks or anticipations, and $1.7 \%$ were discarded due to noise. The remaining $89.5 \%$ of the trials contained valid saccades. Data from one participant were discarded because he tried to guess the location of the eccentric target most of the time, resulting in much higher error rate and a much shorter SRT. Hence, the results are based on the other 15 participants.

Results for the four conditions are shown in Fig. 3. Figure 3a shows the results for the zero-gap interval and Fig. 3b shows the results for the 200-ms gap interval. Each column shows the box plot of a particular combination of the independent parameters, computed using the data from the 15 participants. Each box plot shows the minimum and maximum $S R T$ as the extremes of the dashed line, the lower and upper quartiles as the extremes of the shaded box, the median as a horizontal line within the box, and the mean as a circle ('o'). The values of the mean and one standard deviation are also presented above each box. 


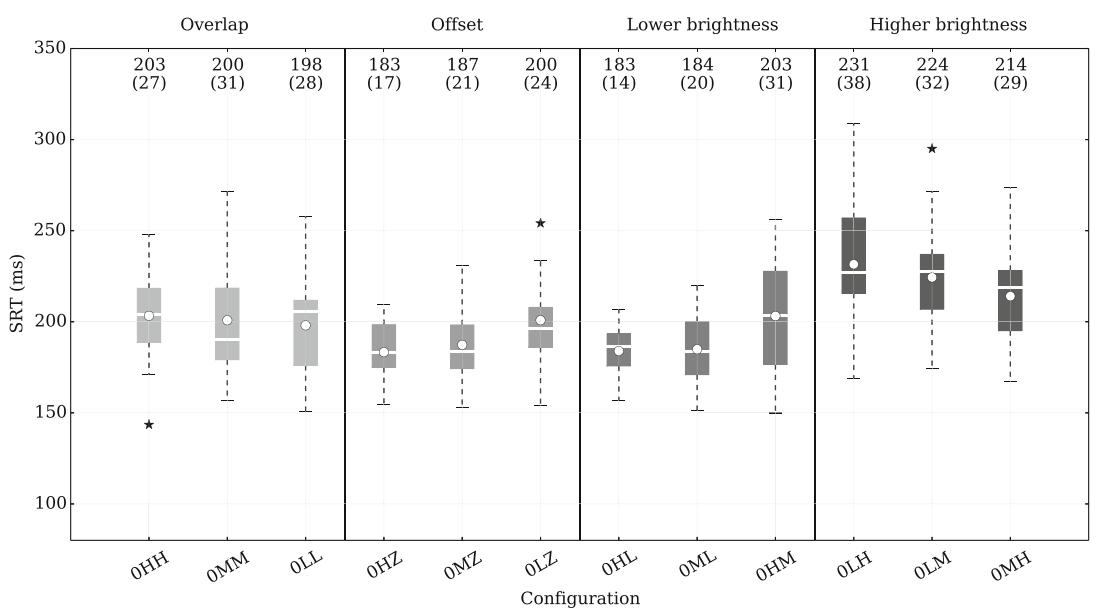

a) Experimental results for the 4 conditions in the zero-gap interval.

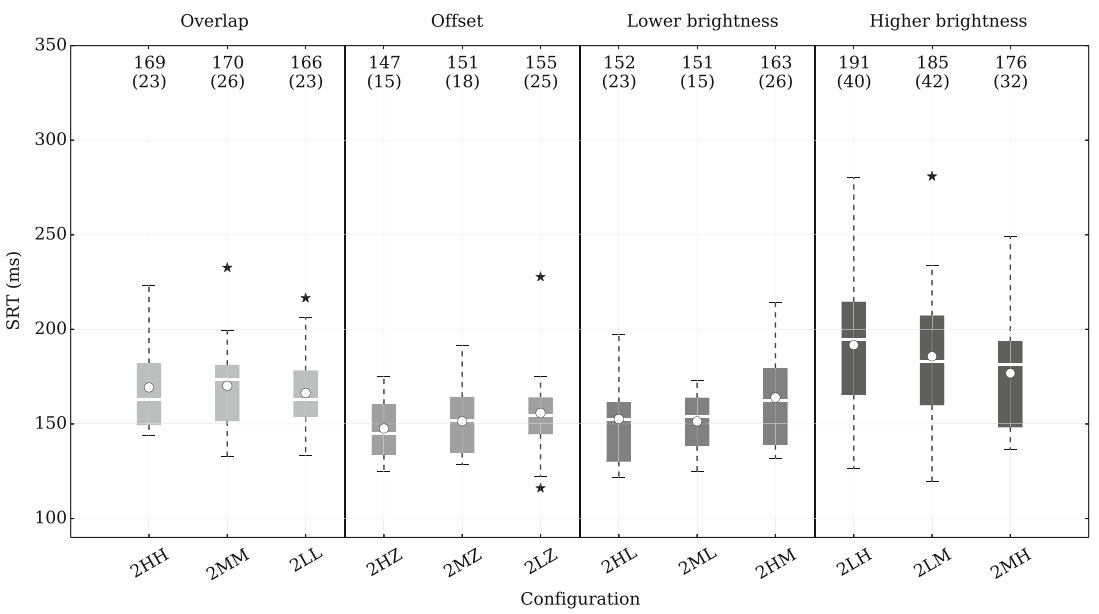

b) Experimental results for the 4 conditions in the $200 \mathrm{~ms}$ gap interval.

Fig. 3 Experimental results for the overlap, offset, lower brightness and higher brightness conditions for 0 - and 200-ms gap intervals. Each visual manipulation was coded using three letters. The first letter indicates a 0 - or 200-ms gap interval, and the second and third letters indicate the brightness transition of the fixation point. Brightness values are coded as H (high), M (medium), L (low), or Z (zero). Each box plot shows the minimum and maximum $S R T$ as the extremes of the dashed line, the lower and upper quartiles as the extremes of the shaded box, the median as a horizontal line within the box, and the mean as a circle ('o'). The values of the mean and one standard deviation are also presented above each box. Outliers are shown as '*'
Outliers are shown as ${ }^{*}$, . Below each box plot there is a three-letter code consistent with the notation introduced in Section "Design", describing gap value and brightness change, defined hereinafter as "configuration".

The rest of this section describes the results of each of the four conditions in turn, and concludes with a global quantitative analysis of all the data based on the model proposed in Eq. 2. Every ANOVA test reported here was preceded by a Mauchly's test of sphericity. In case of sphericity violation, the degrees of freedom and the $p$ value were corrected using the Greenhouse-Geisser correction method, since the estimation of sphericity $(\epsilon)$ was lower than 0.75 in all cases of sphericity violation.

\section{Overlap condition}

In the overlap condition, the fixation point brightness remained high, medium, or low throughout each trial (Fig. 2b). The warning tone was played for $100 \mathrm{~ms}$ and ended at the same time (zero-gap) or $200 \mathrm{~ms}$ before the onset of the eccentric target.

A two-way repeated-measures ANOVA of brightness level (high, medium, low $) \times$ gap $(0,200)$ showed no significant effect of level on $\operatorname{SRT}, F(2,28)=1.56, p=$ $.23, \eta_{p}{ }^{2}=0.1$. Therefore, in the overlap condition, the brightness of the fixation point does not seem to influence the saccadic reaction time. 
Significant changes in $S R T$ were observed as a function of gap, $F(1,14)=66.69, p<.001, \eta_{p}{ }^{2}=0.83$, which can only be attributed to the presence of the warning tone, and was not associated with brightness changes of the fixation point. Using the grand mean of the three brightness levels, SRT went from $201( \pm 29) \mathrm{ms}$ for the zero-gap configurations, to $169( \pm 24) \mathrm{ms}$ for the $200-\mathrm{ms}$ gap configurations, making a difference of about $32 \mathrm{~ms}$. There was no significant interaction between brightness and gap.

\section{Offset condition}

In the offset condition, the fixation point, with initial brightness levels high, medium, or low, was removed at the same time (zero-gap) or $200 \mathrm{~ms}$ (gap) before the onset of the eccentric target. This visual manipulation was synchronized with the warning tone, which was played for $100 \mathrm{~ms}$ and ended at the same time (zero-gap) or $200 \mathrm{~ms}$ before the onset of the eccentric target.

An inverse relationship between the initial brightness level and $S R T$ was found: lower SRT's were associated with higher fixation point levels. A two-way repeatedmeasures ANOVA of brightness (high, medium, low) $x$ gap $(0,200)$ showed a significant main effect of brightness, $F(1.39,19.5)=11.43, p=.0014, \eta_{p}{ }^{2}=0.45$. A post hoc test with Bonferroni correction revealed that $S R T$ for the low level brightness was significantly longer than for the high $(p=.0093)$ and medium $(p=.017)$ levels.

ANOVA also revealed a significant main effect of gap, $F(1,14)=169.24, p<.001, \eta_{p}{ }^{2}=0.92$. A longer grand mean $S R T$ was found for the zero-gap configurations $(190 \pm 22 \mathrm{~ms})$ than for the 200-ms gap configurations (152 $\pm 20 \mathrm{~ms}$ ), a difference of $38 \mathrm{~ms}$. There was no interaction between initial brightness level and gap.

To see whether there was a gap effect due specifically to the removal of the fixation point, $S R T \mathrm{~s}$ in the offset and overlap conditions must be compared, as in Fig. 4.
Considering the high brightness offset (high to zero) and comparing with the control configuration (unchanging level high), for zero-gap there was a significant 20-ms reduction in $S R T, t(14)=5.6, p<.001$, Cohen's $d=1.45$, while for the 200-ms gap, the reduction was $22 \mathrm{~ms}$, also significant, $t(14)=5.99, p<.001$, Cohen's $d=1.55$.

Considering the medium offset (medium to zero) and comparing with the control configuration (level medium), for zero-gap there was a significant 13-ms reduction in $S R T$, $t(14)=4.25, p<.001$, Cohen's $d=1.1$, whereas for the 200-ms gap, the reduction was $19 \mathrm{~ms}$, also significant, $t(14)=5.52, p<.001$, Cohen's $d=1.43$.

When the initial brightness level was low, a significant $S R T$ reduction was observed only for the 200-ms gap, of 11 $\mathrm{ms}, t(14)=3.11, p=.0077$, Cohen's $d=0.8$.

Thus, removing a brighter fixation point produced significant shorter reaction times than removing less-bright fixation points. For both gap intervals we observed a significant gap effect for the high and medium brightness levels, whereas for the low level, the only significant gap effect was for the 200-ms gap. Similar to the overlap condition, the warning tone produced a significant $S R T$ reduction and did not interact with the initial brightness level.

\section{Lower brightness condition}

In the lower brightness condition, the brightness of the fixation point was reduced in the three possible ways: from high to low, medium to low, and high to medium levels at the same time (zero-gap) or $200 \mathrm{~ms}$ before the onset of the eccentric target. Once more, the warning tone was played for $100 \mathrm{~ms}$ and ended at the same time (zero-gap) or $200 \mathrm{~ms}$ before the onset of the eccentric target.

While the results for the high-to-low and medium-to-low configurations were very similar, the high-to-medium configuration presented a longer mean reaction time than the other two. A two-way repeated-measures ANOVA of brightness (high-to-low, medium-to-low, high-to-medium) $\times$ gap

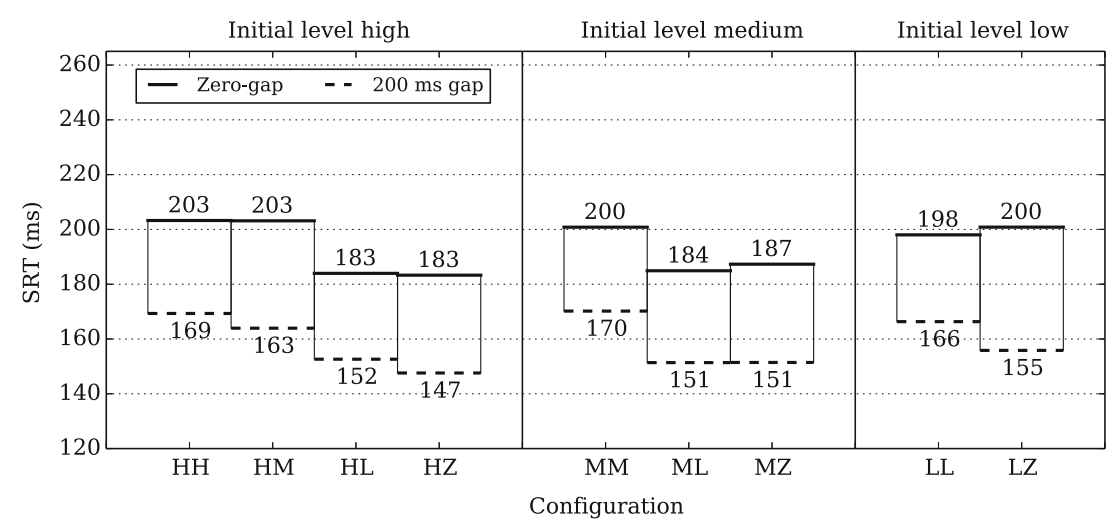

Fig. 4 Grand mean from 15 participants for configurations where the fixation point brightness was reduced for 0- and 200-ms gap intervals, grouped by the initial brightness value 
$(0,200)$ showed a significant main effect of brightness, $F(1.33,18.6)=11.02, p=.002, \eta_{p}{ }^{2}=0.44$. A post hoc test with Bonferroni correction showed that the highto-medium configuration had a significant longer $S R T$ than the high-to-low $(p=.02)$ and medium-to-low $(p=.004)$ configurations.

Analyzing the effect of gap on SRT in this condition, the grand mean for the zero-gap trials $(191 \pm 25 \mathrm{~ms})$ was found to be higher than for the 200-ms gap trials (156 $\pm 23 \mathrm{~ms}$ ), a difference of $35 \mathrm{~ms}$. This difference was significant as revealed by ANOVA, $F(1,14)=85.96, p<$ $.001, \eta_{p}{ }^{2}=0.86$. There was no significant interaction between brightness and gap.

To see whether dimming the fixation point might specifically reduce the $S R T$ in a way comparable to when it is turned off altogether, it is necessary to compare the results of the lower brightness with the overlap condition.

As can be seen in Fig. 4, the high-to-low brightness showed a significant reduction of about $20 \mathrm{~ms}$ for zero-gap, $t(14)=4.28, p<0.001$, Cohen's $d=1.1$, and about $17 \mathrm{~ms}$, also significant, for the 200-ms gap, $t(14)=4.26$, $p<.001$, Cohen's $d=1.1$.

Figure 4 also shows the $S R T$ for the medium-to-low trials. For the zero-gap, there was a significant $S R T$ reduction of about $16 \mathrm{~ms}, t(14)=4.26, p<.001$, Cohen's $d=1.1$, while for the 200-ms gap, the reduction was about $19 \mathrm{~ms}$, also significant, $t(14)=4.14, p=.001$, Cohen's $d=$ 1.06.

However, in the high-to-medium configuration, no significant $S R T$ reduction was observed both for zero-gap, $t(14)=0.04, p=.97$, Cohen's $d=0.01$, and for the 200ms gap, $t(14)=1.06, p=.31$, Cohen's $d=0.27$, as can be observed in Fig. 4.

Summarizing, configurations where the final brightness level was low had significant shorter $S R T$ s than configuration with final level medium, and also produced significant gap effects for both the zero-gap and 200-ms gap, whereas trials where the final brightness was medium did not produce any significant gap effect. Similar to the overlap and offset conditions, the gap interval had a significant effect on SRT and did not interact with the brightness changes.

\section{Higher brightness condition}

In the higher brightness condition, the fixation point brightness was increased in the three possible configurations: lowto-high, low-to-medium, and medium-to-high. This manipulation occurred at the same time (zero-gap) or $200 \mathrm{~ms}$ before the onset of the eccentric target. As in the other three conditions, the warning tone was played for $100 \mathrm{~ms}$ and ended at the same time (zero-gap) or $200 \mathrm{~ms}$ before the onset of the eccentric target.
As can be observed in Fig. 3, SRTs were different among higher brightness configurations. A two-way repeated-measures ANOVA of brightness (low-to-high, low-to-medium, medium-to-high) $\times$ gap $(0,200)$ showed a significant main effect of brightness, $F(1.38,19.3)=7.16$, $p=.009, \eta_{p}{ }^{2}=0.34$. A post hoc test with Bonferroni correction showed that the medium-to-high configuration had a significant shorter $S R T$ than the low-to-high $(p=.006)$ and low-to-medium ( $p=.04)$ configurations.

Analyzing the effect of gap on SRT in this condition, the grand mean for the zero-gap trials $(223 \pm 34 \mathrm{~ms})$ was found to be higher than for the 200-ms gap trials (185 \pm 38 $\mathrm{ms}$ ), a significant difference of $38 \mathrm{~ms}, F(1,14)=22.55$, $p<.001, \eta_{p}{ }^{2}=0.62$. There was no significant interaction between brightness and gap.

To see whether brightening the fixation point might specifically increase the $S R T$ in a way opposite to when it is dimmed or turned off altogether, it is necessary to compare the results of the higher brightness condition with the overlap condition.

As can be seen in Fig. 5, relative to the low-low overlap configuration, the low-to-high configuration showed a significant increase of about $33 \mathrm{~ms}$ for the zero-gap, $t(14)=$ $4.18, p<.001$, Cohen's $d=1.08$, and about $25 \mathrm{~ms}$, also significant, for the 200-ms gap, $t(14)=3.08, p=.008$, Cohen's $d=0.8$.

Figure 5 also shows the SRT for the low-to-medium trials. Relative to the low-low overlap configuration, for zero-gap there was a significant $S R T$ increase of about 26 $\mathrm{ms}, t(14)=3.3, p=.005$, Cohen's $d=0.85$, while for $200 \mathrm{~ms}$ gap the increase was about $19 \mathrm{~ms}$, also significant, $t(14)=2.88, p=.01$, Cohen's $d=0.73$.

In the medium-to-high configuration, also shown in Fig. 5, relative to the medium-medium overlap configuration, a significant $S R T$ increase of $14 \mathrm{~ms}$ was observed for zero-gap, $t(14)=3.1, p=.008$, Cohen's $d=0.43$. For the

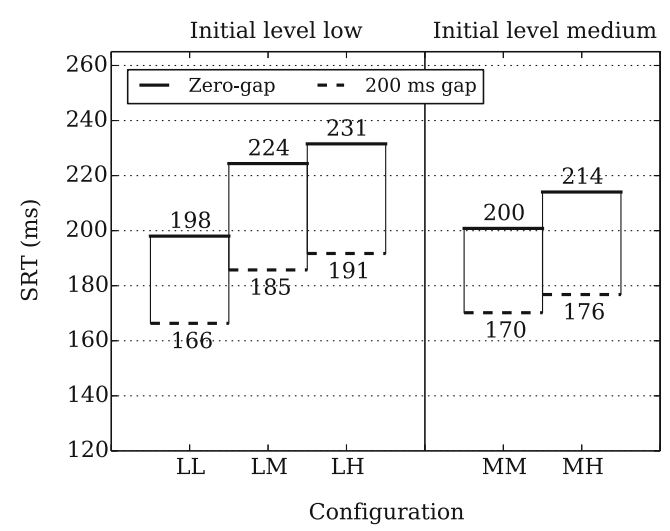

Fig. 5 Grand mean from 15 participants for configurations where the fixation point brightness was increased, for 0- and 200-ms gap intervals, grouped by the initial brightness value 
200-ms gap, a smaller $S R T$ increase of $6 \mathrm{~ms}$ was observed, which was not significant, $t(14)=1.97, p=.07$, Cohen's $d=0.51$.

Results of the higher brightness condition showed that increasing the brightness of the fixation point produces significantly longer reaction times compared with the overlap condition. SRTs were significantly longer in trials with initial brightness level low, compared to trials with initial brightness level medium. Similar to all the other conditions, the gap interval showed a significant effect on SRT's and did not interact with the brightness changes.

\section{Global analysis of the experimental saccadic reaction times}

Recall that our model of saccadic reaction time presented in Eq. 2 has the form:

$$
S R T=\alpha \cdot \log (B G)+\beta
$$

where $\alpha$ is the attentional engagement factor and $\beta$ is the non-modulated reaction time (observed without visual manipulation). Both $\alpha$ and $\beta$ must be determined empirically from experimental data. $B G$ is the brightness gain defined as the ratio of final and initial intensities of the fixation point.

Figure 6 shows the regression results for 0- and 200-ms gap configurations. The horizontal axis at the bottom represents the base $10 \operatorname{logarithm}\left(\log _{10}\right)$ of the brightness gain, and the vertical axis represents the $S R T$ in ms. Each point in the regression corresponds to the mean performance of 15 participants for each experimental configuration. Because for the overlap condition $B G=1$ for all configurations, the grand mean of the three levels (high, medium, and low) was used as a single point for the regression. Thus, the regression was computed using ten points for each gap interval as seen in Fig. 6.

For zero-gap, the proposed model significantly predicts the saccadic reaction time, $F(1,8)=135.96, p<.001$, and $R^{2}=.94$. The estimated value of $\alpha$ was 13.9 $\mathrm{ms} / \log _{10}(\mathrm{BG})$ and had a significant effect, $t(8)=11.66$, $p<.001$, while the estimated value of $\beta$ was $205.1 \mathrm{~ms}$.

For the 200-ms gap, the results also strongly supports the model, $F(1,8)=66.67, p<.001, R^{2}=.89$. The estimated value of $\alpha$ was $12.1 \mathrm{~ms} / \log _{10}(\mathrm{BG})$ and had a significant effect, $t(8)=8.17, p<.001$, while the estimated value of $\beta$ was $167.6 \mathrm{~ms}$.

Because both $\alpha$ values are positive, the curves have positive slopes. The value of $\beta$ can be seen at the points with $\log _{10}(B G)=0$, and corresponds to the $S R T$ for a particular gap interval when there is no visual manipulation.

\section{Discussion}

We first compare our results with existing gap-effect studies in the literature and then discuss the mathematical model

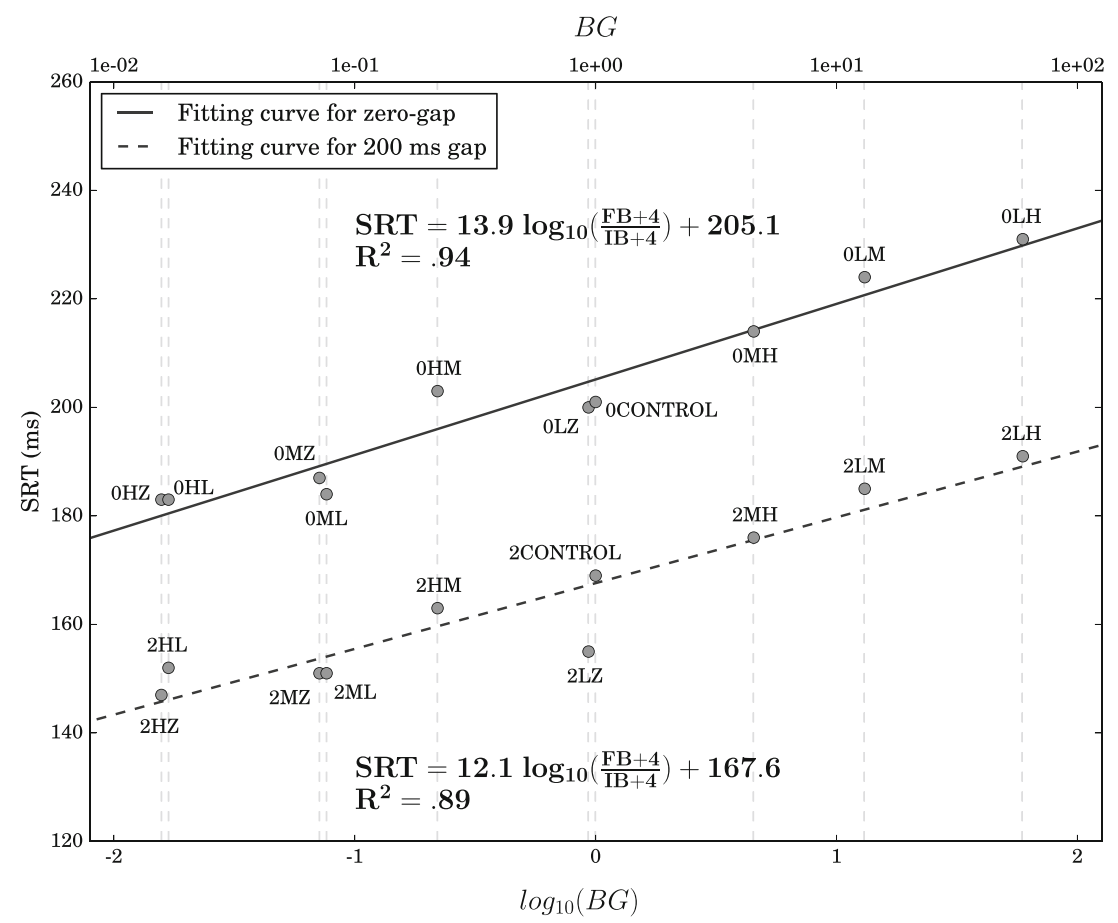

Fig. 6 Results of regression to compute the parameters of the model proposed in Eq. 2 for the 0-and 200-ms gap configurations. Note that the $x$ axis has a logarithmic scale 
of saccadic reaction time based on perceptual brightness changes in the fixation point. Finally, we discuss how the model could be explained by the two current dominant alternative theories of attention disengagement and fixation offset effect.

\section{Comparing with the literature}

In a gap-effect experiment, presenting an auditory warning before the target onset produces an overall reduction in $S R T$ s compared with setups where the auditory warning is not presented. This reduction varies among conditions, being more pronounced in overlap trials because of the lack of visual warning (Pratt et al., 2000; Reuter-Lorenz et al., 1995). Therefore, we must compare our results with other gap-effect studies that also included an auditory warning.

The largest SRT reduction was observed between the high-to-high and high-to-zero configurations for the 200ms gap. This reduction, of about $22 \mathrm{~ms}$, is comparable in magnitude with other gap effect experiments that included a warning tone for gap intervals of $200 \mathrm{~ms}$, such as Pratt et al. (2000) and Reuter-Lorenz et al. (1995).

Since we had two gap intervals ( 0 and $200 \mathrm{~ms}$ ), it is possible to compute a rough estimate of the gap effect we could have observed in our experiment without an auditory warning. Saccadic reaction times in the 200-ms gap trials were on average $36 \mathrm{~ms}$ shorter than in the 0 -gap trials. This reduction due to the gap interval was independent of the brightness changes, since we did not observe any significant interaction between gap values and brightness changes in the ANOVA tests. Given that SRTs are shorter when the auditory warning is synchronized with the target onset than when there is no auditory warning (Ross and Ross, 1981), we would expect to see a gap effect of at least $36+22=58 \mathrm{~ms}$ for the 200-ms gap without an auditory warning, which is compatible with existing studies without an auditory warning, such as Forbes and Klein (1996), Pratt et al. (2000), and Reuter-Lorenz et al. (1995).

Although the experiments of Reulen (1984) did not include an auditory warning, Reulen found an inverse relationship between fixation point brightness and saccadic reaction time. We had three configurations where the fixation point was removed (high-to-zero, medium-tozero, and low-to-zero) and found, similar to Reulen, an inverse relationship between the initial brightness level and SRT's.

Jin and Reeves (2009) also did not include an auditory warning, however we can use the luminance values of their fixation stimulus to estimate the saccadic reaction time difference between the dim and white gap trials according to our model. In the dim gap trial, Jin and Reeves reduced the luminance of the fixation point from $80.1 \mathrm{~cd} / \mathrm{m}^{2}$ to 4.2 $\mathrm{cd} / \mathrm{m}^{2}$, whereas in the white gap trial the luminance was increased from $80.1 \mathrm{~cd} / \mathrm{m}^{2}$ to $117 \mathrm{~cd} / \mathrm{m}^{2}$. Similar to the present experiment, Jin and Reeves presented the stimuli over a black background. The brightness gain for the dim gap trial is $(4.2+4) /(80.1+4)=0.1$, whereas for the white gap trial is $(117+4) /(80.1+4)=1.44$. According to our $S R T$ function for a gap value of $200 \mathrm{~ms}$, the predicted $S R T$ in the dim gap trial is $S R T_{\text {dim }}=12.1 \log _{10}(0.1)+167.6=$ $155 \mathrm{~ms}$, whereas the predicted $S R T$ in the white gap trial is $S R T_{\text {white }}=12.1 \log _{10}(1.44)+167.6=170 \mathrm{~ms}$. Therefore, the predicted difference between the dim and white gap trials is approximately $15 \mathrm{~ms}$. This value is identical to the 15-ms difference found by Jin and Reeves in their randomized design, so our model's prediction was relatively close to the real results, even though there are differences between the experimental setups, like the area and shape of the fixation point, the number of participants and session duration, and the use of an auditory warning.

\section{Model discussion}

Our results showed that saccadic reaction time can be expressed by the mathematical model introduced in Eq. 2 :

$S R T=\alpha \log _{10}(B G)+\beta$

where $B G$ is the brightness gain, defined as the ratio of final and initial brightness of the fixation point (including the ambient luminance), $\alpha$ is the attentional engagement factor, $\beta$ is the non-modulated SRT, and the logarithm is base 10 (without loss of generality).

After fitting the experimental results to the model, we obtained positive values of $\alpha$ for the 0- and 200-ms gap intervals (13.9 and 12.1, respectively). This indicates that increasing the salience of the fixation point elicits longer saccadic reaction times, whereas reducing the salience shortens the saccadic reaction time in an essentially symmetric way.

In the light of our results, we can now answer the following question raised by Reulen (Reulen, 1984): what should the magnitude of the brightness reduction be in order to produce a given gap effect? According to our model, the magnitude of the brightness reduction must be proportional to the initial brightness of the fixation point. More specifically, the final brightness must be ten-times smaller than the initial brightness in order to elicit an approximately 13-ms reduction in $S R T$. Conversely, to increase the $S R T$ by approximately $13 \mathrm{~ms}$, the final brightness must then be ten-times greater than the initial brightness.

Future studies could also include more than one level of background intensity and different eccentricities of the target, since those factors can influence the saccadic reaction time as well. 


\section{Attentional disengagement or FOE}

As pointed out by the reviewers of this paper, this study does not explicitly manipulate attention. Therefore, though our model has been inspired by the thought that the attentional engagement component of the gap effect is modulated by perceptual changes in the fixation point, we acknowledge that the modulation effect reported here could have alternative explanations, such as low-level processes that affect saccadic reaction time. For example, perceptual changes of the fixation point could influence the oculomotor system that controls the execution of saccades in the superior colliculus, since it receives direct input from the retina and the collicular neurons are sensitive to stimulus intensity (Liversedge et al., 2011). If this alternative explanation was valid, the activity of the fixation cells in the rostral pole of the superior colliculus would be modulated by perceptual changes of the fixated stimulus. This hypothesis could be investigated by measuring the activity of the fixation neurons while manipulating the salience of the fixation point, to find out if such correlation exists.

In support of our hypothesis of attentional modulation, our model corroborates that the level of attentional engagement, rather than having two states (released or engaged), varies continuously with perceptual changes of the fixation stimulus. The model shows that the saccadic reaction time is modulated by perceived brightness changes of the fixation point, and can be expressed by the logarithmic model of Eq. 2. Different from Fuller et al. (2009), who found that attentional allocation is modulated by cue contrast, the modulation effect reported in this paper occurs at the fixation point, which the subject is already attending.

Our results could be interpreted as pointing to the existence of both top-down and bottom-up attentional processes that affect the saccadic reaction time. The auditory warning might activate an endogenous process that prepares the disengagement from the fixation point. This top-down disengagement seems to be independent of visual changes in the fixation point, given that the $S R T$ reduction observed from the 0- to 200-ms gap interval was almost constant for all configurations (see Figs. 4 and 5). The bottom-up process is driven by perceptual changes in the fixation point by exogenously releasing or engaging attention accordingly. When the fixation point becomes more salient, attention becomes more engaged, hence delaying the saccadic response. On the other hand, when the fixation point salience is reduced, attention is released, resulting in shorter saccadic reaction times.

Despite possible explanations for the model, quantitative analysis of the gap effect provided by our model has potential value in clinical psychology, both for diagnostic purposes and for cognitive therapy. Other areas such as Human-Computer Interaction could also benefit from the model. For example, Tula et al. (2013) have manipulated the brightness of computer interface elements to improve visual feedback while providing attention disengagement, thus facilitating interaction by exploiting natural eye movements. In this case, quantitative models could be used to dynamically adjust interface parameters, such as contrast, to optimize user response.

\section{Conclusions}

We have presented a mathematical model of saccadic reaction time ( $S R T)$ based on the fixation point brightness gain. The model is based on the Weber-Fechner law, which states that perceived brightness is proportional to the logarithm of the stimulus intensity. It permits to quantity the effect of different brightness manipulations (increasing or reducing the brightness) of the fixation point. We have empirically validated the model using a typical gap-effect experiment with 15 participants, where the brightness gain was manipulated under several conditions, prior to the onset of the eccentric target. Although, in our experiments, we do not directly manipulate attention, we speculate that this modulation effect could be a consequence of attentional engagement being modulated by perceptual changes of the fixation point. Future experiments will be conducted to investigate if these results can actually be attributed to attentional modulation. Nonetheless, because the model can predict $S R T$ changes due to brightness variations, it has potential value in clinical psychology, both for diagnostic purposes and for cognitive therapy.

Acknowledgments This research was supported by the São Paulo Research Foundation (FAPESP), grants 2010/14075-4, 2011/00267-1, and 2012/04426-0. We would like to thank Dr. Walter Machado Pinheiro for several enlightening discussions on the gap effect and on the results reported here.

\section{References}

Awh, E., Belopolsky, A. V., \& Theeuwes, J. (2012). Top-down versus bottom-up attentional control: A failed theoretical dichotomy. Trends in Cognitive Sciences, 16(8), 437-43. doi:10.1016/j.tics. 2012.06.010

Bell, A. H., Meredith, M. A., Van Opstal, A. J., \& Munoz, D. P. (2006). Stimulus intensity modifies saccadic reaction time and visual response latency in the superior colliculus. Experimental Brain Research Experimentelle Hirnforschung. Expérimentation cérébrale, 174(1), 53-59. doi:10.1007/s00221-006-0420-z

Carpenter, R. H. S. (2004). Contrast, probability, and saccadic latency: Evidence for independence of detection and decision. Current Biology, 14(17), 1576-1580. doi:10.1016/j.cub.2004.08.058 
Craig, G., Stelmach, L., \& Tam, W. J. (1999). Control of reflexive and voluntary saccades in the gap effect. Perception \& Psychophysics, 61(5), 935-942. doi:10.3758/BF03206907

Dorris, M. C., \& Munoz, D. P. (1995). A neural correlate for the gap effect on saccadic reaction times in monkey. Journal of Neurophysiology, 73(6), 2558-2562.

Fechner, G. T. (1860). Elemente Der Psychophysik, vol. 2. Leipzig: Breitkopf und Härtel. (reprint 1964. Amsterdam: Bonset).

Fischer, B., \& Breitmeyer, B. (1987). Mechanisms of visual attention revealed by saccadic eye movements. Neuropsychologia, 25(1A), 73-83. doi:10.1016/0028-3932(87)90044-3

Forbes, K., \& Klein, R. M. (1996). The magnitude of the fixation offset effect with endogenously and exogenously controlled saccades. Journal of Cognitive Neuroscience, 8(4), 344-352. doi:10.1162/jocn.1996.8.4.344

Fuchs, I., \& Ansorge, U. (2012). Unconscious cueing via the superior colliculi: Evidence from searching for onset and color targets. Brain Sciences, 2(1), 33-60. doi:10.3390/brainsci2010033

Fuller, S., Park, Y., \& Carrasco, M. (2009). Cue contrast modulates the effects of exogenous attention on appearance. Vision Research, 49(14), 1825-1837. doi:10.1016/j.visres.2009.04.019

Holmqvist, K., Nystrom, M., Andersson, R., Dewhurst, R., Jarodzka, H., \& Van de Weijer, J. (2011). Eye tracking. A comprehensive guide to methods and measures. Oxford: Oxford University Press. ISBN 978-0-19-969708-3.

Jin, Z., \& Reeves, A. (2009). Attentional release in the saccadic gap effect. Vision Research, 49(16), 2045-2055. doi:10.1016/j.visres. 2009.02.015

Kean, M., \& Lambert, A. (2003). The influence of a salience distinction between bilateral cues on the latency of target-detection saccades. British Journal of Psychology (London, England : 1953), 94(Pt 3), 373-388. doi:10.1348/000712603767876280

Kingstone, A., \& Klein, R. M. (1993). Visual offsets facilitate saccadic latency: Does predisengagement of visuospatial attention mediate this gap effect?. Journal of Experimental Psychology: Human Perception and Performance, 19(6), 1251-1265. doi:10.1037/0096-1523.19.6.1251

Liversedge, S., Gilchrist, I., \& Everling, S. (2011). The Oxford handbook of eye movements. Oxford Library of Psychology. New York: OUP Oxford. ISBN 9780199539789.

Mackeben, M., \& Nakayama, K. (1993). Express attentional shifts. Vision Research, 33(1), 85-90. doi:10.1016/0042-6989(93) 90061-Z

Munoz, D. P., \& Wurtz, R. H. (1992). Role of the rostral superior colliculus in active visual fixation and execution of express saccades. Journal of Neurophysiology, 67(4), 1000-1002.

Peirce, J. W. (2009). Generating stimuli for neuroscience using psychopy. Frontiers in Neuroinformatics, 2(10). doi:10.3389/ neuro.11.010.2008
Posner, M. I., \& Petersen, S. E. (1990). The attention system of the human brain. Annual Review of Neuroscience, 13(1), 25-42. doi:10.1146/annurev.ne.13.030190.000325

Pratt, J., Bekkering, H., \& Leung, M. (2000). Estimating the components of the gap effect. Experimental Brain Research, 130, 258-263. doi:10.1007/s002219900243

Pratt, J., Lajonchere, C. M., \& Abrams, R. A. (2006). Attentional modulation of the gap effect. Vision Research, 46(16), 2602-2607. doi:10.1016/j.visres.2006.01.017

Reulen, J. P. H. (1984). Latency of visually evoked saccadic eye movements. II. temporal properties of the facilitation mechanism. Biological Cybernetics, 50(4), 263-271. doi:10.1007/BF00337076

Reuter-Lorenz, P., Hughes, H., \& Fendrich, R. (1991). The reduction of saccadic latency by prior offset of the fixation point: An analysis of the gap effect. Perception \& Psychophysics, 49(2), 167-175. doi:10.3758/BF03205036

Reuter-Lorenz, P. A., Oonk, H. M., Barnes, L. L., \& Hughes, H. C. (1995). Effects of warning signals and fixation point offsets on the latencies of pro-versus antisaccades: Implications for an interpretation of the gap effect. Experimental Brain Research, 103(2), 287-293. doi:10.1007/BF00231715

Ross, S. M., \& Ross, L. E. (1981). Saccade latency and warning signals: Effects of auditory and visual stimulus onset and offset. Perception \& Psychophysics, 29(5), 429-437. doi:10.3758/BF 03207356

Salvucci, D. D., \& Goldberg, J. H. (2000). Identifying fixations and saccades in eye-tracking protocols. In Proceedings of the 2000 Symposium on Eye Tracking Research \& Applications. ETRA '00 (ACM, New York) pp. 71-78. doi:10.1145/355017.355028

Saslow, M. G. (1967). Effects of components of displacement-step stimuli upon latency for saccadic eye movement. Journal of the Optical Society of America, 57(8), 1024-1029. doi:10.1364/ JOSA.57.001024

Taylor, M. J., Carpenter, R. H. S., \& Anderson, A. J. (2006). A noisy transform predicts saccadic and manual reaction times to changes in contrast. The Journal of Physiology, 573(3), 741-751. doi:10.1113/jphysiol.2006.105387

Trappenberg, T. P., Dorris, M. C., Munoz, D. P., \& Klein, R. M. (2001). A model of saccade initiation based on the competitive integration of exogenous and endogenous signals in the superior colliculus. Journal of Cognitive Neuroscience, 13(2), 256-271. doi:10.1162/089892901564306

Tula, A. D., Kurauchi, A. T. N., \& Morimoto, C. H. (2013). Facilitating gaze interaction using the gap and overlap effects. In $\mathrm{CHI}$ ' 13 Extended Abstracts on Human Factors in Computing Systems. CHI EA '13 pp. 91-96. (ACM, New York). ISBN 978-1-4503-1952-2. doi: $10.1145 / 2468356.2468374$ 